\title{
Investigation of the breakpoint region in stacks with a finite number of intrinsic Josephson junctions
}

\section{Shukrinov, Yu M.; Mahfouzi, F.; Pedersen, Niels Falsig}

\section{Published in:}

Physical Review B Condensed Matter

Link to article, DOI:

10.1103/PhysRevB.75.104508

Publication date:

2007

Document Version

Publisher's PDF, also known as Version of record

Link back to DTU Orbit

Citation $(A P A)$ :

Shukrinov, Y. M., Mahfouzi, F., \& Pedersen, N. F. (2007). Investigation of the breakpoint region in stacks with a finite number of intrinsic Josephson junctions. Physical Review B Condensed Matter, 75(10), 104508.

https://doi.org/10.1103/PhysRevB.75.104508

\section{General rights}

Copyright and moral rights for the publications made accessible in the public portal are retained by the authors and/or other copyright owners and it is a condition of accessing publications that users recognise and abide by the legal requirements associated with these rights.

- Users may download and print one copy of any publication from the public portal for the purpose of private study or research.

- You may not further distribute the material or use it for any profit-making activity or commercial gain

- You may freely distribute the URL identifying the publication in the public portal 


\title{
Investigation of the breakpoint region in stacks with a finite number of intrinsic Josephson junctions
}

\author{
Yu. M. Shukrinov, ${ }^{1}$ F. Mahfouzi, ${ }^{2}$ and N. F. Pedersen ${ }^{3}$ \\ ${ }^{1}$ BLTP, JINR, Dubna, Moscow Region, 141980, Russia \\ ${ }^{2}$ Institute for Advanced Studies in Basic Sciences, P. O. Box 45195-1159, Zanjan, Iran \\ ${ }^{3}$ Oersted-DTU, Section of Electric Power Engineering, Technical University of Denmark, DK-2800, Lyngby, Denmark
}

(Received 20 November 2006; revised manuscript received 14 February 2007; published 16 March 2007)

\begin{abstract}
We study the breakpoint region on the outermost branch of the current-voltage characteristics of stacks with different numbers of intrinsic Josephson junctions. We show that at periodic boundary conditions the breakpoint region is absent for stacks with an even number of junctions. For stacks with an odd number of junctions and for stacks with nonperiodic boundary conditions the breakpoint current increases with the number of junctions and saturates at a value corresponding to the periodic boundary conditions. The region of saturation and the saturated value depend on the coupling between the junctions. We explain the results by the parametric resonance at the breakpoint and excitation of a longitudinal plasma wave by Josephson oscillations. A method for diagnostics of the junctions in the stack is proposed.
\end{abstract}

DOI: 10.1103/PhysRevB.75.104508

PACS number(s): 74.25.Fy, 74.78.Fk, 74.81.Fa, 74.40.+k

\section{INTRODUCTION}

A series of experiments devoted to intrinsic Josephson junctions ${ }^{1}$ (IJJs) shows the growing interest in the currentvoltage characteristics (IVC) of a finite stack. ${ }^{2-4}$ Different kinds of couplings between intrinsic Josephson junctions determine the variety of the IVC observed in high-temperature superconductors, and different models are exploited for their descriptions. Among them are the inductive ${ }^{5}$ and capacitive coupling ${ }^{6,7}$ models, which usually give approximately similar results. A unified theory for magnetic and electric coupling in multistacked Josephson junctions was developed. ${ }^{8}$ The capacitively coupled Josephson junction (CCJJ) model seizes the main dynamical properties of the IJJ system, describes the multibranch structure in the IVC of a stack of $\mathrm{IJJs}^{6,9}$ and explains the microwave resonant absorption. ${ }^{10} \mathrm{On}$ the other hand, the diffusion current (DC) plays an important role in a stack of IJJs. ${ }^{11}$ The CCJJ model with diffusion current (CCJJ+DC model) was derived in Ref. 12 on the microscopic level. It gives an equidistant branch structure. ${ }^{13}$ Close to the hysteresis jump the system of IJJs is unstable towards switching. A resonance between the Josephson and plasma oscillations causes the system to switch to another branch. This mechanism in the case of one Josephson junction was considered a long time ago. ${ }^{14}$

A breakpoint region (BPR) on the IVC of a stack of IJJs was demonstrated in Ref. 15 and is explained as a result of resonance between Josephson and plasma oscillations. We consider that simulation of the IVC of IJJs by different groups using different models shows the BPR on the outermost branch as well, but the authors did not mention it [see, particularly, Fig. 3(a) in Ref. 7 in the CCJJ model; Fig. 1 in Ref. 11 in the charge imbalance (CIB) model; Fig. 2 (left) in Ref. 16 in the CIB model]. To our knowledge, no precise experiment to observe the BPR has been carried out yet.

In this paper we show that a detailed investigation of the breakpoint current $I_{b p}$ and BPR width $w_{b p}$ gives us important information concerning the creation of longitudinal plasma waves (LPWs) in stacks of IJJs and the peculiarities of stacks with a finite number of IJJs. We study the IVC of IJJs in the framework of the CCJJ+DC model and investigate the dependence of $I_{b p}$ and $w_{b p}$ on the number of junctions $N$ in the stack. We demonstrate the existence of the BPR on branches corresponding to a stack with one oscillating $(\mathrm{O})$ junction and show that such information may allow one to develop a new method for the diagnostics of the IJJ.

\section{MODEL AND METHOD}

In the CCJJ model ${ }^{6}$ the relation between the charge $\rho_{l}$ and the generalized scalar potential $\Phi_{l}$ of the $l$ th layer is $\rho_{l}=$ $-\left(1 / 4 \pi r_{D}^{2}\right) \Phi_{l}$, where $r_{D}$ is the Debye screening length and $\Phi_{l}$ is expressed through the scalar potential $\phi_{l}$ and the derivative of phase $\theta_{l}$ of the superconducting condensate by $\Phi_{l}(t)=\phi_{l}$ $-(\hbar / 2 e) \partial \theta_{l} / \partial t{ }^{6,11}$ The last relation reflects the nonequilibrium nature of the ac Josephson effect in layered superconductors. ${ }^{11}$ The superconducting layers are in a nonequilibrium state due to the injection of quasiparticles and Cooper pairs. In the equilibrium state $\Phi_{l}(t)=0$. In the $\mathrm{CCJJ}+\mathrm{DC}$ model $^{13}$ with diffusion current $J_{D}^{l}=-\left(\Phi_{l}\right.$ $\left.-\Phi_{l+1}\right) / R$ between layers $l$ and $l+1$ the total external current through the stack has the form

$$
J=C \frac{d V_{l}}{d t}+J_{c}^{l} \sin \left(\varphi_{l}\right)+\frac{\hbar}{2 e R} \dot{\varphi}_{l}
$$

where $V_{l}$ is the voltage between superconducting layers $l$ +1 and $l$ (see below), $\varphi_{l}$ is the gauge-invariant phase difference $\varphi_{l}(t)=\theta_{l+1}(t)-\theta_{l}(t)-(2 e / \hbar) \int_{l}^{l+1} d z A_{z}(z, t)$ between layers $l+1$ and $l, R$ is the junction resistance, and $A_{z}$ is the vector potential in the barrier. This total external current differs from the current in the CCJJ model by the third term in the right-hand side of Eq. (1). In the CCJJ model it is equal to $V_{l} / R$. As a result, in the CCJJ+DC model we obtain the following system of dynamical equations for the phase differences $\varphi_{l}$ : 


$$
\partial^{2} \varphi_{l} / \partial t^{2}=\sum_{l^{\prime}} A_{l l^{\prime}}\left(I-\sin \varphi_{l^{\prime}}-\beta \partial \varphi_{l^{\prime}} / \partial t\right)
$$

with the matrix $A$

$$
A=\left(\begin{array}{ccccccc}
1+\alpha G & -\alpha & 0 & \ldots & & & \\
-\alpha & 1+2 \alpha & -\alpha & 0 & \ldots & & \\
0 & -\alpha & 1+2 \alpha & -\alpha & 0 & \ldots & \\
\vdots & \vdots & \vdots & \vdots & \vdots & \vdots & \vdots \\
& & & \ldots & 0 & -\alpha & 1+\alpha G
\end{array}\right)
$$

where $l^{\prime}$ runs over all $N$ junctions, the parameter $\alpha$ gives the coupling between junctions, $\beta$ is the dissipation parameter $\mathrm{A}\left(\beta^{2}=1 / \beta_{c}\right.$, where $\beta_{c}=\omega_{p}^{2} R^{2} C^{2}$ is the McCumber parameter, $\omega_{p}$ is the plasma frequency, and $C$ is the capacity of the junction), $I$ is the external current normalized to the critical current $I_{c}, G=1+\gamma, \gamma=s / s_{0}=s / s_{N}$, and $s, s_{0}, s_{N}$ are the thicknesses of the middle, first, and last $S$ layers, respectively. In Eq. (2) time is normalized to the plasma frequency $\omega_{p} \cdot{ }^{17}$ According to the proximity effect, we consider that the thicknesses of the first and last layers are different from that of the layers inside the stack. Nonperiodic boundary conditions (BCs) are characterized by the parameter $\gamma$ and the equations for the first and last layers in the system (2) are different from the equation for the middle $S$ layer. ${ }^{6,17}$ For periodic BCs the matrix $A$ has the form

$$
A=\left(\begin{array}{ccccccc}
1+2 \alpha & -\alpha & 0 & \ldots & & & -\alpha \\
-\alpha & 1+2 \alpha & -\alpha & 0 & \ldots & & \\
0 & -\alpha & 1+2 \alpha & -\alpha & 0 & \ldots & \\
\vdots & \vdots & \vdots & \vdots & \vdots & \vdots & \vdots \\
-\alpha & & & \ldots & 0 & -\alpha & 1+2 \alpha
\end{array}\right)
$$

We solve this system for stacks with different numbers $N$ of intrinsic junctions. The numerical procedure has been done as follows. For a given set of model parameters $N, \alpha, \beta, \gamma$ we simulate the IVC of the system, i.e., $V_{l}(I)$, increasing $I$ from zero up and then down. A change in the parameters $N, \alpha, \beta, \gamma$ changes the branch structure in the IVC essentially. Their influence on the IVC in the CCJJ and CCJJ+DC models was discussed in Refs. 9, 13, and 17. To calculate the voltages $V_{l}(I)$ in each point of the IVC (for each value of $I$ ), we simulate the dynamics of the phases $\varphi_{l}(t)$ by solving the system of equations (2) using the fourth-order Runge-Kutta method. After simulation of the phase dynamics we calculate the dc voltages on each junction as

$$
\partial \varphi_{l} / \partial t=\sum_{l^{\prime}} A_{l l^{\prime}} V_{l^{\prime}}
$$

where $V_{l}$ is normalized to $V_{0}=\hbar \omega_{p} /(2 e)$. The average of the voltage $\bar{V}_{l}$ is given by

$$
\bar{V}_{l}=\frac{1}{T_{\max }-T_{\min }} \int_{T_{\min }}^{T_{\max }} V_{l} d t,
$$

where $T_{\min }$ and $T_{\max }$ determine the interval for the averaging. After completing the voltage averaging for current $I$, the current $I$ is increased or decreased by a small amount $\delta I$ to calculate the voltages at the next point of the IVC. We use the distribution of phases and their derivatives achieved in the previous point of the IVC as the initial distribution for the current point.

Numerical stability was checked by doubling and dividing in half the temporal discretization steps $D t$ and checking the influence on the IVC. Finally we can obtain the total dc voltage $V$ of the stack by

$$
V=\sum_{l=1}^{N} \bar{V}_{l} .
$$

At some current $I$ some junction (or junctions) switches to the nonzero-voltage state and gives some branch of the IVC. We plot the total IVC at different parameters of the problem. 

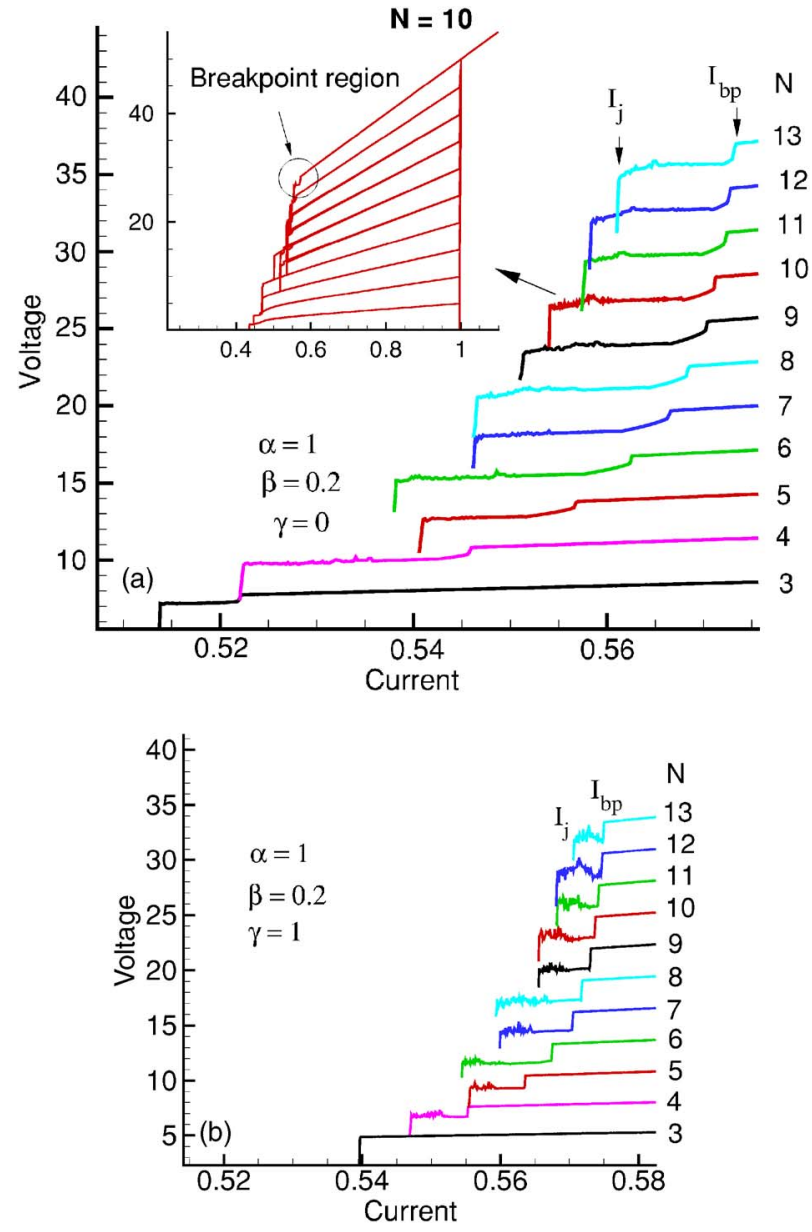

FIG. 1. (Color online) IVC of the outermost branch for stacks with different numbers $N$ of IJJs at $\gamma=$ (a) 0 and (b) 1 .

The details concerning the numerical procedure are given in Refs. 7 and 17.

To investigate the BPR in detail, we have calculated the IVC for different boundary conditions for stacks with different numbers $N$ of IJJs from $N=3$ to 30 . For clarity, we restrict the number of curves in some figures.

\section{IVC FOR STACKS WITH DIFFERENT NUMBERS OF IJJs}

The result of simulation of the total branch structure in the IVC for a stack of ten IJJs in the CCJJ+DC model by Eq. (2) is presented in the inset of Fig. 1(a). As we can see, this IVC demonstrates the BPR on the outermost branch.

The outermost branch corresponds to the state of the stack with all junctions in the rotating (R) state, ${ }^{17}$ and it is the upper branch in the IVC. The values of the breakpoint current $I_{b p}$ and transition current $I_{j}$ (the jumping point to the next branch in the IVC) on the outermost branch are shown by arrows in Fig. 1(a). The distance between these two values we call the width $w_{b p}$ of the BPR. We have found that the breakpoint current $I_{b p}$ and BPR width $w_{b p}$ depend on the parameters $\alpha$ and $\beta$, the boundary conditions, and the number of junctions in the stack.

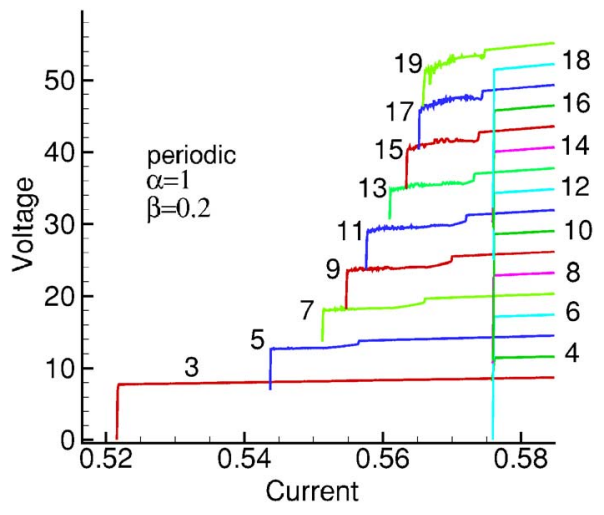

FIG. 2. (Color online) IVC of the outermost branch for stacks with different numbers of junctions at periodic BCs.

Let us first describe the main features of the BPR which follow from the results of the simulation. As we can see in Fig. 1(a), at $\gamma=0$ both $I_{b p}$ and $I_{j}$ increase with $N$, but the increase of $I_{b p}$ is monotonic. The IVC of the stacks with even $N$ has larger $w_{b p}$ at small $N$. The IVC in the BPR shows a chaotic behavior, and its width is decreased with $N$. There is a saturation of $N$ dependence of the $I_{b p}$ at large $N$.

At $\gamma=1$ [Fig. 1(b)] these features remain unchanged but the value of $w_{b p}$ is decreased for all stacks, especially for $N=4$, and it is equal to zero for $N=3$. This change in the boundary conditions leads to a relative change of the BPR width in different stacks as well.

The IVC with periodic boundary conditions (Fig. 2) shows the same behavior for the $I_{b p}$ and BPR width $w_{b p}$ for the stacks with odd $N$ as in the nonperiodic case, but for the stacks with even $N$ the value of $I_{b p}$ does not depend on $N$ and the BPR for these stacks is absent.

In Fig. 3(a) the $I_{b p}$ as a function of $N$ for $\gamma=0$ (squares, curve 1) and periodic (circles, curve 2) boundary conditions at different values of the coupling parameter $\alpha$ is presented. We stress the coincidence of the $N$ dependencies of $I_{b p}$ for stacks with odd numbers of IJJs for the periodic and $\gamma=0$ cases. The increase in $\alpha$ leads to the saturation of the $N$ dependence at larger $N$. The value of the saturated $I_{b p}$ decreases with decreasing coupling and is 0.576 at $\alpha=1,0.454$ at $\alpha=0.5$, and 0.304 at $\alpha=0.1$. At $\alpha=0$ the breakpoint coincides with the return current, so $I_{b p}$ has the same value for stacks with different numbers of junctions.

The $N$ dependence of the BPR width $w_{b p}$ for stacks with even and odd numbers of junctions at $\gamma=0$ (curves $1_{\text {even }}$ and $1_{\text {odd }}$ ) and periodic (curve $2_{\text {odd }}$ ) boundary conditions is shown in Fig. 3(b). The main feature here is a decrease of the BPR width $w_{b p}$ with increasing $N$ at large $N$. At small $N$ in the interval $(3,6)$ we observe an increase of $w_{b p}$ with $N$.

\section{THE ORIGIN OF THE BREAKPOINT ON THE OUTERMOST BRANCH}

To explain the observed features of the finite-stack IVC let us discuss the origin of the breakpoint on the outermost branch. The hysteresis jump in the IVC is associated with a change of the distribution pattern of rotating phase 

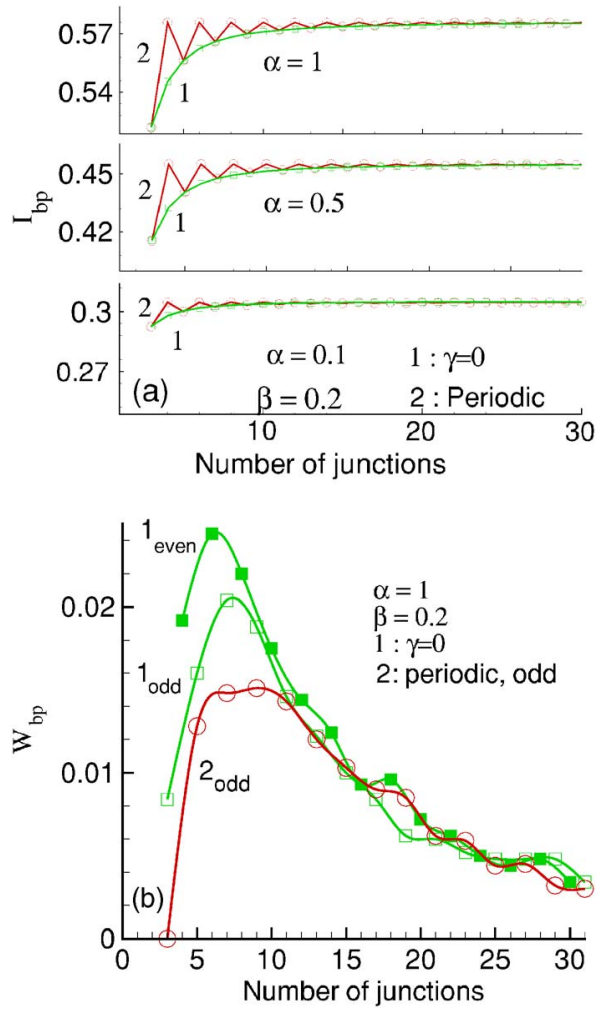

FIG. 3. (Color online) (a) $N$ dependence of $I_{b p}$ for $\gamma=0$ (curve 1) and periodic (curve 2) boundary conditions at different $\alpha$; (b) $N$ dependence of the BPR width $w_{b p}$ for stacks with even and odd numbers of junctions at $\gamma=0$ (1) and periodic (2) boundary conditions.

motions. ${ }^{17}$ But the question of why a change in the current leads to a change in the distribution pattern of the rotating phase motions is still open. We consider the case that all junctions are in the rotating state, i.e., the time average of $\bar{\varphi}_{l}$ $\left\{\bar{\varphi}_{l}=\left[1 /\left(T_{\max }-T_{\min }\right)\right] \int_{T_{\min }}^{T_{\max }} \varphi_{l} d t\right\}$ is constant and that of $\sin \varphi_{l}$ is zero for these junctions. For oscillating junctions the situation is opposite: the time average of $\bar{\varphi}_{l}$ is zero and that of $\sin \varphi_{l}$ is constant.

As we mentioned above, the outermost branch in the IVC corresponds to the state of the stack with all junctions in the rotating state. Let us write an equation for the difference of the phase differences $\delta_{l}=\varphi_{l+1}-\varphi_{l}$ for the outermost branch.

By subtracting Eq. (2) for the (l)th from that for the $(l$ $+1)$ th junction we get

$$
\begin{aligned}
& \left(\ddot{\varphi}_{l+1}-\ddot{\varphi}_{l}\right)+\left(1-\alpha \nabla^{(2)}\right)\left[\sin \left(\varphi_{l+1}\right)-\sin \left(\varphi_{l}\right)+\beta\left(\dot{\varphi}_{l+1}-\dot{\varphi}_{l}\right)\right] \\
& \quad=0 .
\end{aligned}
$$

Here $\nabla^{(2)} f_{l}=f_{l+1}+f_{l-1}-2 f_{l}$ is the discrete Laplacian. Consider the linear approximation $\sin \left(\varphi_{l+1}\right)-\sin \left(\varphi_{l}\right) \approx \delta_{l} \cos (\varphi)$, where $\varphi \simeq \Omega t=(1 / N) V t, \Omega$ is the Josephson frequency, and $V$ is the total voltage of the stack, we obtain

$$
\ddot{\delta}_{l}+\left(1-\alpha \nabla^{(2)}\right)\left[\cos (\varphi) \delta_{l}+\beta \dot{\delta}_{l}\right]=0 .
$$

Expanding $\delta_{l}(t)$ in the Fourier series
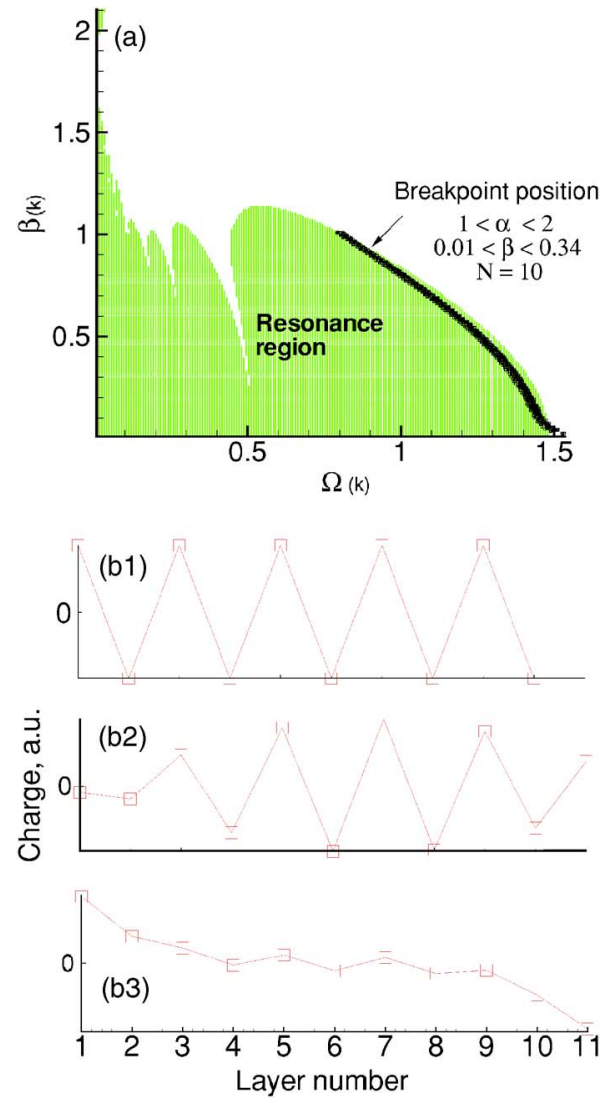

FIG. 4. (Color online) (a) Parametric resonance region in $\Omega(k)$ $-\beta(k)$ diagram. Black dots (stripe) correspond to the breakpoint current $I_{b p}$ in the IVC for $k=\pi$ at different values of parameters $\alpha$ and $\beta$. (b) Charge on the $\mathrm{S}$ layers in the stacks with 10 (b1) and 11 (b2) IJJs at periodic BCs and with 10 IJJs at $\gamma=1$ (b3).

$$
\delta_{l}(t)=\sum_{k} \delta_{k} e^{i k l},
$$

the linearized equation for the Fourier component of the difference of the phase differences $\delta_{k}$ between neighbor junctions can be written in the form ${ }^{15}$

$$
\ddot{\delta}_{k}+\beta(k) \dot{\delta_{k}}+\cos [\Omega(k) \tau] \delta_{k}=0,
$$

where $\tau=\omega_{p}(k) t, \omega_{p}(k)=\omega_{p} C_{\alpha}, \beta(k)=\beta C_{\alpha}, \Omega(k)=\Omega / C_{\alpha}$, and $C_{\alpha}=\sqrt{1+2 \alpha[1-\cos (k)]}$.

The important fact for us is that this linearized equation manifests a parametric resonance in the system of IJJs. In Fig. 4(a) we plot the resonance region for this equation on the $\beta(k)-\Omega(k)$ diagram. The dark stripe on this figure is actually the distribution of the dots, corresponding to the positions of the breakpoints of the outermost branch. Using the breakpoint values of the voltage in the equation $\Omega(k)$ $=\Omega / C_{\alpha}=V / N C_{\alpha}$, we obtain this distribution of the breakpoints by the variation of the coupling parameter $\alpha$ in the interval $(1,2)$ with a step 0.1 and the dissipation parameter $\beta$ in the interval $(0.01,0.34)$ with the step 0.01 at each value of $\alpha$. In contrast to the results presented in Ref. 15 where the positions of the dots on the $\beta(k)-\Omega(k)$ diagram were obtained by crude estimation, here we have done precise nu- 
merical calculations. These calculations show closer positions of the breakpoints to the boundary of the resonance region in the chosen intervals of $\alpha$ and $\beta$. The reason that the positions of the breakpoints are shifted from the boundary of the resonance region in Fig. 4(a), is the linear approximation used to obtain Eq. (9).

The breakpoints are inside the resonance region, i.e., the resonance between the Josephson and plasma oscillations is approached at the breakpoint $I_{b p}$. As a result, the plasma mode is excited by the Josephson oscillations. We can prove this statement directly. By the Maxwell equation $\operatorname{div}(E / d)$ $=4 \pi \rho$, we express the charge $\rho_{l}$ on the superconducting $(\mathrm{S})$ layer $l$ by the voltages $V_{l, l-1}$ and $V_{l, l+1}$ in the neighboring insulating layers $\rho_{l}=\left(\epsilon_{0} / 4 \pi d_{0} d\right)\left(V_{l, l+1}-V_{l-1, l}\right)$. The time dependence of $\rho_{l}$, presented in Fig. 4(b1), demonstrates that with periodic BCs in the stacks with 10 IJJs a LPW with $k$ $=\pi$ is realized. Really, the charge on the nearest-neighbor layers has the same value and opposite sign. Figure 4(b2) shows the distribution of the charge on the $\mathrm{S}$ layers in the stacks with 11 IJJs with periodic BCs. In this case we observe the creation of a LPW with $k=10 \pi / 11$. To determine the mode of the LPW with nonperiodic BCs, we need more detail investigation. Figure 4(b3) demonstrates the charge distribution in the stack with 10 IJJs at $\gamma=1$ near the breakpoint.

The wavelengths of the standing LPWs that can be realized in a stack with $N$ junctions are $N / n$ lattice units in the $z$ direction, where $n$ changes from 1 to $N / 2$ for stacks with an even number of junctions and from 1 to $(N-1) / 2$ for odd $N$. The voltage of the stack at the breakpoint is related to the wave number $k$ of the LPW by the formula $V$ $=N \Omega(k) \sqrt{1+2 \alpha[1-\cos (k)]}$, so the largest breakpoint voltage $V$ in the current-decreasing process corresponds to the creation of a LPW with $k$ equal to $\pi$ ( $\pi$ mode) for stacks with even numbers of IJJs and modes with $k=(N-1) \pi / N$ for stacks with odd $N$.

\section{DISCUSSION OF THE MAIN RESULTS}

Let us now return to the results presented in Figs. 1 and 3 and demonstrate that they are in agreement with the ideas stated in the previous section.

According to these ideas, in the stack with ten IJJs a LPW with $k=(N-1) \pi / N$ is created and it leads to an increase of $I_{b p}$ with $N$ and its saturation to a value corresponding to the $\pi$ mode. The same modes are created in the stacks with $\gamma$ $=1$ for which the outermost branches of the IVC are presented in Fig. 1(b). They demonstrate the saturation of $I_{b p}$ at the same value.

As Fig. 2 shows, with periodic BCs we observe the same value of $I_{b p}$ in all the stacks with even $N$. This is in agreement with our suggestion that in this case a LPW with $k$ $=\pi$ is created. We check it directly as well, by the time dependence of $\rho_{l}$. We find that at periodic BCs in the stacks with even $N$ the charge on the nearest-neighbor layers has the same value and opposite sign, which means that a LPW with $k=\pi$ is realized.

In the stacks with odd $N$ the $\pi$ mode cannot exist, so as we mentioned in the previous section, a LPW with the largest $k$ equal to $(N-1) \pi / N$ is created. The creation of different modes of the LPW leads to different $I_{b p}$, and this fact explains the difference in the IVC with periodic BCs of the stacks with even and odd numbers of IJJs. With increase in $N$ the wave number $k$ reaches the limiting value $\pi$, and it leads to the increase in $I_{b p}$ that we observe in Fig. 2.

It explains as well the saturation of $I_{b p}$ to the value corresponding to the $I_{b p}$ for stacks with even $N$ that is demonstrated in Fig. 3(a).

The difference between the charge distribution on the $\mathrm{S}$ layers at the breakpoint current at fixed time for stacks with even and odd numbers of IJJs (10 and 11) with periodic BCs is demonstrated in Fig. 4(b). In the stack with odd $N$ the charges on the first and last layers oscillate in phase and the oscillations on the neighbor $\mathrm{S}$ layers are different from the oscillations in the $\pi$ mode. Because at $\gamma=0$ the charge on the first and last layers is screened due to the proximity effect, a LPW with $k=(N-1) \pi / N$, as with periodic BCs for stacks with odd $N$, is also created for stacks with both odd and even $N$. This is the reason the values of $I_{b p}$ for stacks with odd $N$ coincide for both these BCs as shown in Fig. 3(a).

At $\gamma=1$ the same modes are created, but the character of the charge distribution among the $\mathrm{S}$ layers is different [as we can see in Fig. 4(b3)]. As a result, in this case the $N$ dependence of $I_{b p}$ is stronger than at $\gamma=0$, but at $N \rightarrow \infty$ it is saturated as well and the saturation value is the $I_{b p}$ for even junctions with periodic BCs.

The influence of the coupling parameter on the value of the breakpoint current $I_{b p}$ and its $N$ dependence, which is demonstrated in Fig. 3(a), has a clear explanation. It consists in the $\alpha$ dependence of the voltage as mentioned in the previous section, and correspondingly the breakpoint current $I_{b p}$. They are proportional to the term $\left.\sqrt{1+2 \alpha[1-\cos (k)}\right]$. An increase in $k$ leads to a term proportional to $\sqrt{1+4 \alpha}$. The decrease in $\alpha$ makes this influence weaker.

We may explain the increase in $w_{b p}$ at small $N$, which is shown in Fig. 3(b), by a commensurability effect on the width of the BPR. With periodic BCs $w_{b p}=0$, if the wavelength $\lambda$ of the LPW is $\lambda=n$, where $n=2,3,4, \ldots$ lattice units in the $z$ direction. As we can see in Fig. 2, at $\lambda=3$ (for stacks with $N=3)$ and $\lambda=2(\pi$ mode), the IVC do not show the BPR. The creation of the LPW with wavelength in the middle of the interval $(2,3)$ should correspond to some maximum of the BPR width $w_{b p}$. In a stack with five IJJs, the LPW with $\lambda=2.5(k=4 \pi / 5)$ is created. It does not coincide exactly with the result obtained by the simulation, because we use for the explanation the linearized equation for the difference of phase differences and Fourier expansion in the finite stacks. The wave number $k$ is not well defined in this case. With increase in $N$ the wave numberk of the LPW limits to $\pi$ and this explains the increase in $I_{b p}$ and the decrease in $w_{b p}$ at large $N$, which is demonstrated in Fig. 3(b). The change in the boundary conditions changes the character of the charge oscillations on the S layers. In particular, at $\gamma$ $=1$ we observe a decrease in $w_{b p}$ for all stacks. Our analysis shows that in stacks with even $N$ at nonperiodic BCs the charges on the second and $N-1$ layers oscillate in phase, but antiphase in the stacks with odd $N$. We consider that the value of the BPR width $w_{b p}$ depends on the character of the charge oscillations in the $S$ layers. 


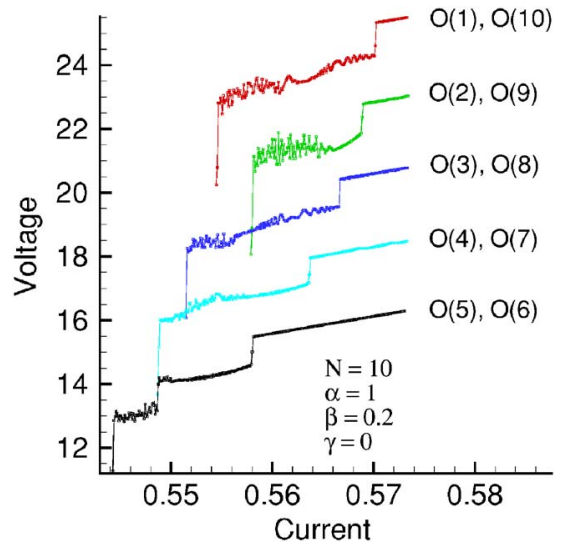

FIG. 5. (Color online) BPR on the branches of the IVC of stacks with one oscillating junction in the case of ten IJJs at $\alpha=1, \beta$ $=0.2$, and $\gamma=0$. The top curve corresponds to the real scale of voltage, but the others are shifted down for clarity by two units.

Finally, we note that in the case of coupling between junctions the parameter $\beta$ cannot be determined in the usual way by the return current, because it depends now on two parameters $\beta$ and $\alpha$. The dependence of $I_{b p}$ and the BPR width $w_{b p}$ on the dissipation and coupling parameters opens an opportunity to develop a new method for determination of these parameters for stacks of IJJs. This question will be discussed in detail elsewhere.

\section{ONE OSCILLATING JUNCTION}

Let us now discuss briefly the breakpoints on the other branches of the IVC.

As we mentioned above, the resistive state in the system of IJJs is realized as a state with different numbers of $\mathrm{R}$ and O junctions. ${ }^{9,17}$ The different positions of $\mathrm{R}$ and $\mathrm{O}$ junctions in the stack (different configurations) correspond to different states of the IJJ system. Equidistant positions of the $\mathrm{O}$ junction from the ends of the stack (for example, stacks with a first or tenth $\mathrm{O}$ junction) lead to the same state. So there are five different states in the stack with one $\mathrm{O}$ junction corresponding to the different position of this junction. Figure 5 shows the BPR on the branches of the IVC of stacks with one $\mathrm{O}$ junction in the case of ten IJJs at $\alpha=1, \beta=0.2$, and $\gamma=0$.

Equidistant positions of the $\mathrm{O}$ junction from the ends of the stack lead to the same value of $I_{b p}$ and the same width of the BPR. The shift of the $\mathrm{O}$ junction from the end of the stack to its center decreases the $I_{b p}$ of the corresponding state. So we may establish a delay of LPW creation in the current-decreasing process when the position of the $\mathrm{O}$ junction is shifted to the center of the stack.

We consider that the origin of such behavior is as follows. This one oscillating junction separates the stack into two parts with different numbers of $\mathrm{R}$ junctions which are weakly coupled through it. With a decrease in current the first LPW is created in the part with the largest $I_{b p}$ (with the largest number of junctions). The shift of the $\mathrm{O}$ junction and the decrease in the number of $\mathrm{R}$ junctions in this part lead to a decrease of $I_{b p}$ as Fig. 1 demonstrates. The increase of the number of junctions in the second part might manifest a second breakpoint which is related to the creation of a LPW in this second part of the stack. This situation is observed for $N=10$ when the $\mathrm{O}$ junction occupies the fifth or sixth site in the stack. The width of the BPR in the other branches of the IVC depends essentially on the state of the stack.

For the other branches the increase in the number of $\mathrm{O}$ junctions in the stack decreases the number of effective junctions for creation of the LPW and leads to a decrease of the return current. This fact explains why we can obtain a total branch structure in the hysteresis region, because in the other case we would not be able to observe it in the simulation. The correspondence between the position of the $\mathrm{O}$ junction in the stack and the value of $I_{b p}$ opens the possibility for junction diagnostics, i.e., by measuring the value of $I_{b p}$ we can answer the question of which junction in the stack goes into the $\mathrm{R}$ or $\mathrm{O}$ state. From the other side, the monitoring of the transitions between branches is useful for understanding the phase dynamics in a system of IJJs.

\section{CONCLUSIONS}

In conclusion, we stress that the BPR in the IVC naturally follows from the solution of the system of dynamical equations for the phase difference for a stack of IJJs. In the breakpoint region the plasma mode is a stationary solution of the system and this fact might be used in some applications, particularly in high-frequency devices such as terahertz oscillators and mixers. The detailed study of the breakpoint current and breakpoint region width gives a new opportunity for the investigation of the properties of IJJs and for development of new methods for determination of the parameters of IJJs and diagnostics of IJJS in stacks.

\section{ACKNOWLEDGMENTS}

We thank P. Müller, R. Kleiner, A. Ustinov, T. Koyama, M. Machida, A. Yurgens, Yu. Latyshev, A. Irie, M. Sargolzaei, T. Boyadjiev, N. M. Plakida, Y. Sobouti, and M. R. $\mathrm{H}$. Khajehpour for stimulating discussions and support of this work.
${ }^{1}$ R. Kleiner, F. Steinmeyer, G. Kunkel, and P. Muller, Phys. Rev. Lett. 68, 2394 (1992); G. Oya, N. Aoyama, A. Irie, S. Kishida, and H. Tokutaka, Jpn. J. Appl. Phys., Part 2 31, L829 (1992).
${ }^{2}$ Myung-Ho Bae, Jae-Hyun Choi, and Hu-Jong Lee, cond-mat/ 0610334 (unpublished).

${ }^{3}$ L. X. You, M. Torstensson, A. Yurgens, D. Winkler, C. T. Lin, and B. Liang, Appl. Phys. Lett. 88, 222501 (2006). 
${ }^{4}$ Y.-J. Doh, H.-J. Lee, and H.-S. Chang, Phys. Rev. B 61, 3620 (2000); Ch. Preis, Ch. Helm, K. Schmalzl, J. Keller, R. Kleiner, and P. Müller, Physica C 362, 51 (2001).

${ }^{5}$ S. Sakai, P. Bodin, and N. F. Pedersen, J. Appl. Phys. 73, 2411 (1993).

${ }^{6}$ T. Koyama and M. Tachiki, Phys. Rev. B 54, 16183 (1996).

${ }^{7}$ M. Machida, T. Koyama, and M. Tachiki, Phys. Rev. Lett. 83, 4618 (1999).

${ }^{8}$ M. Machida and S. Sakai, Phys. Rev. B 70, 144520 (2004).

${ }^{9}$ Yu. M. Shukrinov and F. Mahfouzi, Physica C 434, 6 (2006).

${ }^{10}$ Y. Matsuda, M. B. Gaifullin, K. Kumagai, K. Kadowaki, and T. Mochiku, Phys. Rev. Lett. 75, 4512 (1995).

${ }^{11}$ D. A. Ryndyk, Phys. Rev. Lett. 80, 3376 (1998).
${ }^{12}$ M. Machida, T. Koyama, A. Tanaka, and M. Tachiki, Physica C 330, 85 (2000).

${ }^{13}$ Yu. M. Shukrinov, F. Mahfouzi, and P. Seidel, Physica C 449, 62 (2006).

${ }^{14}$ N. F. Pedersen, J. Mygind, O. H. Soerensen, and B. Dueholm, J. Phys. Colloq. 6, 1232 (1978); H. H. Zappe, J. Appl. Phys. 44, 1371 (1973).

${ }^{15}$ Yu. M. Shukrinov and F. Mahfouzi, Semicond. Sci. Technol. 20, S38 (2007).

${ }^{16}$ Yu. M. Shukrinov and F. Mahfouzi, J. Phys.: Conf. Ser. 43, 1143 (2006).

${ }^{17}$ H. Matsumoto, S. Sakamoto, F. Wajima, T. Koyama, and M. Machida, Phys. Rev. B 60, 3666 (1999). 